\title{
Resultate der Urabstimmung der FMH Services Genossenschaft
}

\section{Beat Bumbachera, Beat Bär ${ }^{b}$}

${ }^{a}$ Dr. med., MLaw Präsident der Verwaltung FMH Services Genossenschaft; ${ }^{b}$ Geschäftsführer FMH Services Genossenschaft

\section{Geschätzte Genossenschafterinnen und Genossenschafter}

Mit dem Geschäftsbericht haben wir Sie im Mai 2019 über unsere operativen Aktivitäten informiert und Sie um Genehmigung des Geschäftsberichts 2018, der Zuweisung des Resultats und die Entlastung der Verwaltung gebeten. Zudem stand die Wahl von Herrn Dr. med. Simone Ghisla als neues Mitglied in die Verwaltung und die Wahl von vier bisherigen Verwaltungsräten an.

Wir freuen uns, Ihnen nun die Resultate der Urabstimmung präsentieren zu können, und danken Ihnen sehr für Ihre Teilnahme und Ihr Interesse.

Freundliche Grüsse

FMH Services Genossenschaft

\section{Urabstimmung 2019}

\section{Stimmabgabe}

17. Mai-28. Juni 2019 schriftlich bei der Gemeinde Oberkirch

\section{Auszählung}

Herr J. R. Rogger, Gemeindeverwaltung Oberkirch

\section{Resultate}

\subsection{Antrag 1}

Genehmigen Sie den Geschäftsbericht der FMH Services 2018?

\begin{tabular}{lr}
\hline Abgegebene Stimmen & 3710 \\
\hline Ungültige Stimmen (keine Unterschrift) & 13 \\
\hline Leere und keine Stimmen & 64 \\
\hline Gültige Stimmen & 3633 \\
\hline JA-Stimmen & $\mathbf{3 5 3 7}$ \\
\hline NEIN-Stimmen & $\mathbf{9 6}$ \\
\hline
\end{tabular}

Dem Antrag 1 wird somit deutlich zugestimmt.

\subsection{Antrag 2}

Sind Sie einverstanden, den Verlust von CHF 388171.93 auf die neue Rechnung vorzutragen und mit den bestehenden Reserven zu verrechnen?

\begin{tabular}{lr}
\hline Abgegebene Stimmen & 3712 \\
\hline Ungültige Stimmen (keine Unterschrift) & 13 \\
\hline Leere und keine Stimmen & 93 \\
\hline Gültige Stimmen & 3606 \\
\hline JA-Stimmen & $\mathbf{3 3 6 8}$ \\
\hline NEIN-Stimmen & $\mathbf{2 3 8}$
\end{tabular}

Dem Antrag 2 wird somit deutlich zugestimmt.

\subsection{Antrag 3}

Erteilen Sie der Verwaltung der FMH Services Genossenschaft für das Berichtsjahr 2018 Entlastung?

\begin{tabular}{lr}
\hline Abgegebene Stimmen & 3710 \\
\hline Ungültige Stimmen (keine Unterschrift) & 13 \\
\hline Leere und keine Stimmen & 101 \\
\hline Gültige Stimmen & 3596 \\
\hline JA-Stimmen & 3381 \\
\hline NEIN-Stimmen & $\mathbf{2 1 5}$
\end{tabular}

Dem Antrag 3 wird somit deutlich zugestimmt. 


\subsection{Antrag 4}

Stimmen Sie der Wahl der Revisionsstelle Truvag Revisions AG in Sursee für eine weitere Amtsperiode von einem Jahr zu?

\begin{tabular}{lr}
\hline Abgegebene Stimmen & 3713 \\
\hline Ungültige Stimmen (keine Unterschrift) & 13 \\
\hline Leere und keine Stimmen & 79 \\
\hline Gültige Stimmen & 3621 \\
\hline JA-Stimmen & 3519 \\
\hline NEIN-Stimmen & 102
\end{tabular}

Dem Antrag 4 wird somit deutlich zugestimmt.

\subsection{Antrag 5}

Stimmen Sie der Wahl der Gemeindeverwaltung Oberkirch als neutraler Drittstelle zur Abwicklung der Urabstimmung für eine weitere Amtsperiode von einem Jahr zu?

\begin{tabular}{lr}
\hline Abgegebene Stimmen & 3711 \\
\hline Ungültige Stimmen (keine Unterschrift) & 13 \\
\hline Leere und keine Stimmen & 178 \\
\hline Gültige Stimmen & 3520 \\
\hline JA-Stimmen & 3471 \\
\hline NEIN-Stimmen & 49
\end{tabular}

Dem Antrag 5 wird somit deutlich zugestimmt.

\subsection{Antrag 6}

Stimmen Sie der Wahl von Herrn Dr. med. Simone Ghisla als neuem Mitglied der Verwaltung zu?

\begin{tabular}{lr}
\hline Abgegebene Stimmen & 3704 \\
\hline Ungültige Stimmen (keine Unterschrift) & 13 \\
\hline Leere und keine Stimmen & 240 \\
\hline Gültige Stimmen & 3451 \\
\hline JA-Stimmen & 3333 \\
\hline NEIN-Stimmen & 118
\end{tabular}

Dem Antrag 6 wird somit deutlich zugestimmt.

\subsection{Antrag 7}

Stimmen Sie der Wahl von Herrn Dr. med. Beat Bumbacher (bisher) als Präsident der Verwaltung zu?

\begin{tabular}{lr}
\hline Abgegebene Stimmen & 3708 \\
\hline Ungültige Stimmen (keine Unterschrift) & 13 \\
\hline Leere und keine Stimmen & 91 \\
\hline Gültige Stimmen & 3604 \\
\hline JA-Stimmen & 3466 \\
\hline NEIN-Stimmen & 138 \\
\hline
\end{tabular}

Dem Antrag 7 wird somit deutlich zugestimmt.

\subsection{Antrag 8}

Stimmen Sie der Wahl von Herrn Dr. rer. publ. HSG Sven Bradke (bisher) als Vizepräsident der Verwaltung $\mathrm{zu}$ ?

\begin{tabular}{lr}
\hline Abgegebene Stimmen & 3705 \\
\hline Ungültige Stimmen (keine Unterschrift) & 13 \\
\hline Leere und keine Stimmen & 101 \\
\hline Gültige Stimmen & 3591 \\
\hline JA-Stimmen & 3428 \\
\hline NEIN-Stimmen & 163 \\
\hline
\end{tabular}

Dem Antrag 8 wird somit deutlich zugestimmt.

\subsection{Antrag 9}

Stimmen Sie der Wahl von Herrn Dr. med. Pierre Sindelar (bisher) als Mitglied der Verwaltung zu?

\begin{tabular}{lr}
\hline Abgegebene Stimmen & 3706 \\
\hline Ungültige Stimmen (keine Unterschrift) & 13 \\
\hline Leere und keine Stimmen & 97 \\
\hline Gültige Stimmen & 3596 \\
\hline JA-Stimmen & $\mathbf{3 4 7 1}$ \\
\hline NEIN-Stimmen & $\mathbf{1 2 5}$
\end{tabular}

Dem Antrag 9 wird somit deutlich zugestimmt.

\subsection{Antrag 10}

Stimmen Sie der Wahl von Herrn Dr. med. Remo Osterwalder (bisher) als Mitglied der Verwaltung zu?

\begin{tabular}{lr}
\hline Abgegebene Stimmen & 3706 \\
\hline Ungültige Stimmen (keine Unterschrift) & 13 \\
\hline Leere und keine Stimmen & 87 \\
\hline Gültige Stimmen & 3606 \\
\hline JA-Stimmen & 3478 \\
\hline NEIN-Stimmen & 128
\end{tabular}

Dem Antrag 10 wird somit deutlich zugestimmt.

Das Ergebnis dieser Urabstimmung wurde von J. R. Rogger, c/o Gemeindeverwaltung Oberkirch, am 5. Juli 2019 als vollständig und richtig bestätigt. 